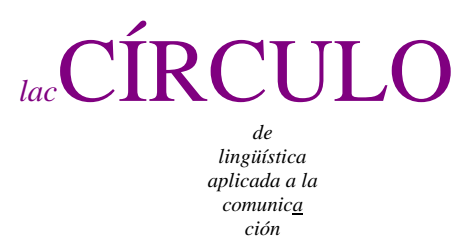

$68 / 2016$

\title{
INTERRELACIÓN ENTRE VOCALIZACIONES Y DESARROLLO PROSÓDICO EN FUNCIONES COMUNICATIVAS TEMPRANAS
}

\author{
Asier Romero Andonegi \\ Universidad del País Vasco/ Euskal Herriko Unibertsitatea \\ a romero en ehu es
}

Resumen

Este estudio explora la intención comunicativa en la relación entre las vocalizaciones y la prosodia en la fase lingüística de una sola palabra. El corpus de análisis lo han constituido los actos comunicativos de seis niños y niñas vascos de entre $1 ; 1$ y 1;8 y con lengua materna euskera. El análisis se ha centrado en la producción acústica sobre un total de 675 vocalizaciones extraídos de aproximadamente 3 h. de grabación en casa de los informantes. Los datos extraídos de las grabaciones muestran que (1) las vocalizaciones comunicativas y no comunicativas no se diferencian de forma significativa a partir de su contorno entonativo final, (2) las funciones comunicativas tempranas se diferencian por su contorno entonativo final, destacando las funciones imperativas y fáticas por su asociación con las tonalidades ascendentes.

Asier Romero Andonegi. 2016.

Interrelación entre vocalizaciones y desarrollo prosódico en funciones comunicativas tempranas.

Círculo de Lingüística Aplicada a la Comunicación 68, 350-379.

http://www.ucm.es/info/circulo/no68/romero.pdf

http://revistas.ucm.es/index.php/CLAC

http://dx.doi.org/10.5209/CLAC.54534

(C) 2016 Asier Romero Andonegi.

Círculo de Lingüística Aplicada a la Comunicación (clac)

Universidad Complutense de Madrid. ISSN 1576-4737. http://www.ucm.es/info/circulo 
Palabras clave: Gestos tempranos, prosodia, adquisición del lenguaje, intencionalidad comunicativa, función comunicativa, vasco

\begin{abstract}
Interrelation between vocalization and prosodic development in early communication functions. This study explores the communicative intention in the relationship between vocalizations and linguistic prosody in the transition to first words. The corpus of analysis has been built taken into account six Basque children at age 1;8 and 1;8 being Basque the mother tongue. The analysis has focused on the vocal production of a total of 675 vocalizations extracted from approximately $3 \mathrm{~h}$ of at-home made recording. The extracted data shows that (1) communicative and non-communicative vocalizations are not significantly different based on pitch final contour, (2) early communicative functions can be differentiated based on their final pitch contour, especially the imperative and phatic functions by association with the rising final pitch contour.

Key words: communicative function, prosody, communicative function, language acquisition, basque
\end{abstract}

Índice

1. Introducción 352

1.1. Intención comunicativa y función comunicativa 355

1.2. Adquisición de la competencia prosódica 357

2. Corpus y metodología 361

2.1. Participantes 361

2.2. Procedimiento 361

3. Resultados de la investigación 368

4. Conclusiones 371

Bibliografía 374 


\section{Introducción}

Los niños son capaces de comunicarse a través de varias modalidades o conductas comunicativas (coordinación de miradas con su interlocutor, gestos motores, vocalizaciones) o con la coordinación de dos o más de estas modalidades. Así, coordinando conductas comunicativas los niños consiguen una mayor claridad y refuerzan su mensaje, ya que una de las conductas coordinadas puede reforzar o aclarar la función comunicativa de la otra. En este contexto, Sarriá (1991) propone que estas conductas presentan una cierta organización, al existir un elemento máximo y constante, que constituye el núcleo del acto comunicativo, y diversos elementos variables, de menor duración en sus apariciones, que se vinculan con el elemento máximo. Por tanto, la actividad temprana de los niños incluye, tanto conductas vocales, como no-vocales.

Si atendemos a la definición que aporta López Ornat y Karousou (2005) sobre las vocalizaciones, estas serían producciones tempranas interpretables por los padres, con o sin contenido segmental, en las que el niño produce grupos prosódicos de duración variable. Así, no estaríamos, pues, ante una unidad léxica definida, con contenido fonológico convencional, sino, más bien, de una aproximación a la palabra adulta que, aunque inmadura aún, exhibe un carácter tanto comunicativo como prosódico que la dota de sentido para los padres, los principales interlocutores del niño en edades tempranas. Estas producciones constituyen uno de los primeros pasos en la progresión hacia la adquisición completa del lenguaje. Una manifestación que se va a ir poniendo en un lugar preeminente conforme el adulto le vaya concediendo, casi exclusivamente, el carácter de instrumento para regular su actividad, compartir estados mentales, percibirse como emisor y receptor, etc.

En esta investigación, se propone que la coordinación gestual y las vocalizaciones encierran las bases de la asociación entre forma prosódica y función comunicativa. Hay que tener en cuenta que los componentes de “forma” y “función” que caracterizan al lenguaje en general se hallan también en las vocalizaciones. Más aún, según resultados de investigación evolutiva, esos componentes de las vocalizaciones evolucionan hasta converger en los valores convencionales de la lengua ambiental. Esta asociación permitiría al niño, en momentos tempranos, comunicarse de forma eficiente con sus interlocutores habituales. Posteriormente, el niño irá adquiriendo asociaciones más complejas, como la asociación entre forma segmental y el significado léxico que 
subyace a la adquisición de las palabras convencionales de tipo adulto, o los aspectos intencionales y referenciales necesarios que se irán haciendo cada vez más comunes en el uso lingüístico infantil.

El estudio de las vocalizaciones prelingüísticas no ha recibido mucha atención por parte de los especialistas de la adquisición del lenguaje sino hasta hace relativamente pocos años. El infrecuente contenido segmental de las vocalizaciones y su complejidad prosódica, las hacen difíciles de transcribir, codificar, medir e interpretar. Además, estas producciones resultan inmaduras en términos fonológicos y altamente dependientes del contexto (Nathami y Oller, 2001). En los últimos años, diversos investigadores (Karousou, 2003; Oller, 2000; Vihman, 1996) han desarrollado sistemas de codificación fiables que pueden simplificar y agilizar el registro y análisis de datos de la actividad vocal anterior a las primeras palabras.

A esta dificultad transcriptora hay que unir razonamientos teóricos. Uno de ellos podría ser la influencia de las teorías innatistas, cuya posición frente a los datos de producción ha sido siempre muy escéptica. Otra, probablemente haya sido que tanto la neuropsicología clásica como la actual, evolutiva o no, han desarrollado mucho más trabajo sobre la relación del hemisferio izquierdo con los procesos lingüísticos, que sobre su relación con el hemisferio derecho. Así el componente “derecho” de las vocalizaciones (entonación, comprensión, por ejemplo) las ha podido alejar de la mirada neurocientífica (López Ornat y Karousou, 2005).

Por tanto, a tenor de la evidencia que se constata en la literatura relacionada con la adquisición del lenguaje, se puede señalar que en el temprano proceso de adquisición lingüística y, que el niño tempranamente va desarrollando, éste logra comunicarse de forma eficaz por medio de vocalizaciones, gestos y miradas (en combinación con el surgimiento de intenciones comunicativas aproximadamente desde los 10 meses, Bates et al. 1975). Las intenciones o funciones comunicativas son transmitidas por los niños por medio de una protopalabra o una vocalización, es decir, por medio de un patrón prosódico más o menos definido aunque carente de contenido léxico o segmental convencional (Vihman, 1994). En la evolución de la expresión de las intenciones comunicativas infantiles interviene, de una forma clara, el progresivo dominio de componentes formales de la lengua, como el patrón acentual, los distintos contornos tonales, etc. Así, el aprendizaje de la lengua supondría desarrollos diversos que se entrecruzan por momentos, aunque progrese cada uno a su propio ritmo (Karousou, 2003). 
En resumen, la continuidad del crecimiento lingüístico se establece a partir de investigaciones detalladas que muestran cómo las formas vocalizadas por los bebés, y sus funciones, acercan la producción del aprendiz de lengua cada vez más a las formas de sus lengua(s) ambientale(s). Además, múltiples estudios detallan cómo los componentes prosódico, articulatorio, segmental y comunicativo de las vocalizaciones se acercan lenta y progresivamente a esos mismos valores de la(s) lengua(s) modelo (de Boysson-Bardies et al 1981; D’Odorico, 1984; D’Odorico \& Franco, 1991; Elbers, 1982; Halliday, 1982; Koopmans-van Beinum \& van der Stelt, 1986; Oller, 2000; Oller et al. 1976).

La presente investigación muestra que, entre los 13 y 20 meses, los bebés con lengua materna vasca son ya capaces de producir signos lingüísticos basados en una asociación entre aspectos prosódicos, y funciones o intenciones comunicativas. Así, atendiendo a contorno entonativo final no es posible determinar la diferenciación entre las vocalizaciones comunicativas y no comunicativas. Además, se muestra que las diferentes funciones comunicativas analizadas en el estudio se relacionan significativamente con los aspectos prosódicos de contorno entonativo final. En ese sentido, se muestra que las primeras asociaciones entre forma y significado que realizan los infantes se dan entre los aspectos prosódicos de la emisión y las funciones comunicativas o pragmáticas específicas.

El artículo se divide en cinco secciones. Después de la introducción, en la primera sección se realiza un repaso de las nociones de intención comunicativa y función comunicativa, junto con su relación con la adquisición de la competencia prosódica. En la segunda sección, se presenta la metodología seguida en la presente investigación, para lo cual se describe el corpus de participantes, el procedimiento y la codificación adoptada en este estudio. En la tercera sección, por un lado, se presentan los resultados relacionados con la función del contorno entonativo final en el contraste de vocalizaciones comunicativas y no comunicativas; y, por otro lado, en el contraste entre las distintas funciones comunicativas analizadas. Por último, en la cuarta sección, se presentan y discuten las principales conclusiones obtenidas a lo largo de la investigación. 


\subsection{Intención comunicativa y función comunicativa}

La combinación de gesto-habla es un rasgo fundamental de la condición humana. Como señala McNeill (1992) en el lenguaje gestual humano las modalidades discursivas son coordinadas no sólo en el nivel fonológico sino que también en los niveles semánticos y pragmáticos. Así, los niños comienzan a usar combinaciones intencionadas de gestohabla cerca del final del primer año de vida, unos meses después de iniciado el balbuceo canónico y precediendo a la etapa lingüística de la primera palabra. (Butcher \& GoldinMeadow, 2000; Esteve-Gibert \& Prieto, 2014; Murillo \& Capilla, 2015).

La presencia de estos comportamientos combinados ha sido interpretada como un indicador de comunicación intencional; y aunque existe un acuerdo bastante generalizado en la literatura sobre la edad de aparición de la conducta intencional, se observan discrepancias a la hora de invocar las causas subyacentes que posibilitan la aparición de dicha conducta. Algunos autores relacionan los orígenes de la comunicación intencional con el desarrollo cognoscitivo (Bates, Camaioni \& Volterra, 1975; Piaget, 1936). Otros insisten en la importancia de la interacción social entre el bebé y el adulto, como hecho determinante en la emergencia de la comunicación intencional (Kaye, 1982; Vygotsky, 1979). Finalmente, otras explicaciones se basan en la existencia de una motivación innata en los bebés para comunicarse con el entorno (Blomm, 1993; Trevarthen, 1979). Ahora bien, la investigación sigue siendo necesaria; por ejemplo, en relación con el rol que adquiere la función pragmática en las combinaciones gesto-habla en contextos socio-comunicativos específicos, y su valor potencial predictivo en el posterior desarrollo del lenguaje (Igualada, Bosch \& Prieto, 2015).

El descubrimiento por parte del niño de que sus propias vocalizaciones pueden servirle como medio para dirigir la atención del otro y ejercer una influencia en su conducta supone un paso esencial en la adquisición del lenguaje. Es en este periodo, cuando el niño descubre el sentido y la función del lenguaje; es decir, sus signos le permiten expresar intenciones y, en esa medida mover a los demás a actuar de forma determinada.

De acuerdo con Bates (1975), las primeras intenciones comunicativas aparecen hacia los 10 meses de edad y con una característica fundamental, dejan en este punto de ser meramente perlocutivas para devenir ilocutivas. Así, estas vocalizaciones pasan a ser realmente intencionales, es decir, a estar movidas por una intención del emisor, lo que se evidencia a través de indicios como la persistencia del niño en su conducta hasta lograr el efecto deseado o la reformulación de la misma cuando el intento inicial no logra resultados. 
Tanto Bates (1975) como Dore (1975), señalan la capacidad del niño de dominar las funciones pragmáticas del lenguaje antes de haber adquirido las estructuras de frase propias de su lengua materna. A partir de los 24 meses, esta circunstancia evoluciona ya que los niños producen tanto vocalizaciones que tienen intención comunicativa como otras que no la tienen. En este contexto, es fundamental determinar si se trata de un tipo de emisiones u otro. Distintos autores han investigado al respecto, así para Coggins et al. (1981) consideran que una secuencia conductual producida por el niño encierra una intención comunicativa cuando este se encuentra participando de forma conjunta con su interlocutor en una actividad compartida. Sarriá (1991) propone la noción de acto comunicativo intencional preverbal como grupo de conductas que en conjunto poseen la capacidad efectiva de transmitir un mensaje, y que son realizadas por un sujeto/emisor de forma voluntaria con ese fin, bien siendo el único objetivo o integrado en un conjunto de varios objetivos.

En conclusión, podemos decir que la intencionalidad comunicativa se construye en el curso de la interacción niño-adulto y se manifiesta por medio de una emisión vocal orientada a un destinatario con el objetivo de influir en él. En la presente investigación, se considera que una vocalización tiene intención comunicativa cuando se produce en el marco de la interacción o produce por efecto un encuentro interaccional nuevo y transmite, conjuntamente, un determinado mensaje al interlocutor, que reacciona en consecuencia. Por tanto, la intencionalidad se origina en el deseo del niño de influir en el interlocutor de una forma determinada y se completa en el momento en el que el interlocutor interpreta la producción vocal del niño y actúa de forma correspondiente, lógicamente asumiendo el carácter intencional de la expresión infantil.

En la investigación, se consideraron solo aquellas conductas que involucran un componente vocal y no aquellas compuestas exclusivamente de gestos, aunque como es lógico no se excluye la concurrencia de gestos y vocalizaciones. En este contexto, la acción del niño fue considerada como conducta intencionada comunicativa cuando se observaron los siguientes indicios, (a) el niño orienta su mirada hacia el adulto interlocutor, actual o potencial, mientras vocaliza; (b) el niño proporciona al adulto un tiempo para intervenir después de haber vocalizado; (c) el adulto se siente interpelado por la vocalización infantil y, en esa medida, responde o comenta la intervención vocal 
del niño; (d) el niño manifiesta su frustración cuando, aparentemente, la reacción del interlocutor no le satisface y modifica su emisión vocal en consecuencia.

\subsection{Adquisición de la competencia prosódica}

El acto de habla primitivo propuesto por Dore (1974) esta formado por una “protopalabra(s)” o patrón prosódico y su función es transmitir la intención del niño en un momento en que no es capaz de producir oraciones completas. El desempeño fonológico resulta aún inmaduro pero los patrones prosódicos empleados, acompañados o no de un contenido léxico, transmiten la fuerza ilocutiva del acto de habla al indicar al interlocutor cómo pretende el niño que sea entendida su vocalización, o cuál es su intención comunicativa (Boysson-Bardies \& Vihman, 1991). En esta línea, Vihman (1996) ya señala que los sonidos presentes en este tipo de vocalizaciones iniciales aparecen después, también, en las primeras palabras. A partir de los 12 meses el niño desarrolla la capacidad de producir vocalizaciones cada vez más semejantes al habla de tipo adulto. Por tanto, se observa una relación de continuidad entre las formas propias del balbuceo variado y aquellas más cercanas al lenguaje convencional de tipo adulto.

Con todo, hay que señalar que a la hora de la evaluación del desarrollo lingüístico, quizá esté influyendo el hecho de que, a fin de cuentas, las vocalizaciones -pero no los gestospresentan también continuidad de forma con el habla futura. Es decir, tanto la función comunicativa como la convencionalización progresiva (simbolización), pueden desarrollarse de modo continuo desde gestos y vocalizaciones hasta el lenguaje organizado. Pero en esta evolución sólo las vocalizaciones presentan, además, continuidad formal con el habla (Butcher y Goldin-Meadow, 2000, Esteve-Gibert y Prieto, 2014 y Romero et al., 2015).

Algunas investigaciones han demostrado que los niños perciben la prosodia dentro del vientre materno, y que con cinco meses son capaces de diferenciar la lengua materna de las demás por medio del ritmo (Peña-Garay, 2005); otras, que los niños adquieren la entonación antes de decir las primeras palabras (Bever, et al. 1971; Locke, 1983). Cortés (2002) señala que la adquisición de la entonación ocurre de manera progresiva, ya que los entornos comunicativos de los niños son escasos y se sigue adquiriendo la entonación incluso después de la pubertad. Baeza-Álvarez, desde un punto de vista 
comunicativo, subrayan la importancia tanto de la percepción como del uso en un contexto adecuado:

El análisis se refiere a la asignación del significado de la información que entra a través de la percepción y por último a dicho significado se le dará uso en el contexto adecuado. La comprensión se produce como resultado de la interacción entre la información obtenida (escrita o hablada) y los conocimientos previos del oyente o lector, dependiendo del caso. (Baeza-Álvarez, 2011: 17)

Otros autores han propuesto el componente prosódico como facilitador no sólo para la gramática, sino también para el léxico en el aprendizaje del lenguaje especialmente en sus inicios; e incluso llegan a proponer un bootstrapping relativo a este nivel (Morgan y Demuth, 1996; Jusczyk, 1997; López-Ornat, 1999). Además, estudios recientes han confirmado que los niños producen una amplia variedad de acentos tonales específicos del idioma y combinaciones de tono de frontera antes del inicio del periodo de las dos palabras. Estos datos dan argumentos a la hipótesis de que estos niños tenían un importante conocimiento de la entonación gramatical antes de la producción de sus primeras dos palabras; y arroja dudas sobre la hipótesis de que el desarrollo de la gramática de los niños coincide en el tiempo con el desarrollo de la entonación, sugiriendo por tanto que el desarrollo gramatical entonativo es previo al desarrollo gramatical (Prieto \& Vanrell, 2007; Frota \& Vigário, 2008).

Por otra parte, dentro de las teorías tradicionales del desarrollo fonológico se encuentra la teoría prosódica (Waterson 1981). En ella se propone que los niños no perciben los segmentos fonéticos al detalle, sino que durante un buen tiempo desarrollan las habilidades perceptivas y productivas a partir de la melodía, y muchas de las unidades fonéticas son realizadas de forma no analizada (no contrastada). Progresivamente, los niños van atendiendo a las unidades contrastables perceptivamente, como son la acentuación, la silabificación y, finalmente, los fonemas y los rasgos. El hecho de poner en el centro del aprendizaje la oración o la palabra ha tenido ventajas que posteriormente la fonología no lineal ha retomado. Con esta aproximación, que tiene en cuenta la palabra como unidad (no la de tipo adulto sino tal como puede ser formada por el niño), se pueden explicar muchas simplificaciones o errores que no son claros ni sistemáticos cuando se analizan desde una perspectiva en la cual el fonema o la sílaba son las unidades de procesamiento. Así el fonema no es la unidad básica, sino que es 
contingente al lugar que ocupa en la palabra: según sea percibido se organiza su representación y su producción. Una explicación de este tipo tiene la ventaja de incluir la diversidad individual, la variación entre contextos léxicos, y de otorgar un gran peso al input que reciben los niños y a la percepción que éstos realizan. Sin embargo, no llega a explicar fenómenos complejos que se encuentran más allá del aprendizaje inicial, ni tampoco las constantes entre los sujetos, hechos que ciertamente parecen depender de la estructura fonológica y de sus contrastes, independientemente de otros contextos más amplios.

La lógica dificultad que entraña el aprendizaje del inventario fonológico de la lengua por parte del niño, le posibilita, en unos primeros momentos, de los aspectos suprasegmentales para poder expresarse y comunicarse. Lieberman (1984) sostiene que, debido al rápido descenso en la presión subglotal hacia el fin del grupo respiratorio, existe una tendencia universal que motiva que los valores de frecuencia fundamental $\left(\mathrm{F}_{0}\right)$ e intensidad declinen hacia el final de la emisión. Esta circunstancia ha posibilitado que se considere el tono descendente como el patrón por defecto en la entonación. Por tanto, este tono se caracterizaría por requerir de un menor esfuerzo y, por ello, el más común en las vocalizaciones infantiles al tener una base fisiológica. Sin embargo, para Snow y Balog (2002), y Snow (2004) los tonos descendentes también pueden ser producto de la experiencia infantil con la lengua materna, y aunque los tonos ascendentes requerirían de una mayor esfuerzo que los descendentes para la mayoría de los hablantes, ambos necesitan la participación de mecanismos activos de producción de habla.

Vihman (1996) argumenta con dos posibles razones para justificar la adquisición del elemento suprasegmental (prosódico) antes que el segmental (fonológico). En primer lugar, los rasgos prosódicos resultarían llamativos para los niños desde casi un inicio y, a la vez, parecen susceptibles de manipulación voluntaria en la producción temprana. En segundo lugar, invariablemente el número de patrones prosódicos es menor que el de los segmentos. Esto explicaría la capacidad del niño tan sólo de dominar una parte del inventario adulto de fonemas, mientras que para ese mismo periodo de una palabra sería capaz de producir vocalmente las características prosódicas del sistema adulto.

Diversas investigaciones han descrito evidencias de una asociación temprana entre prosodia y función comunicativa: relacionando registros tonos ascendentes con 
funciones imperativas (o con demanda de algún tipo de intervención), vocalizaciones que expresan funciones comunicativas con menor duración y valores de frecuencia fundamental más bajos que aquellas que expresan solo emociones o diferencias de patrón prosódico y duración entre las vocalizaciones comunicativas y no comunicativas (D’Odorico \& Franco, 1991; Flax et al., 1991; Papaeliou et al. 2002; Fernández, 2014). Pero, ¿cómo aprendemos a pronunciar, a acentuar o a entonar en nuestra L1 o lengua nativa? En el caso de la pronunciación los diferentes autores coinciden en resaltar la importancia de la imitación de los niños en sus interlocutores. Ahora bien, la repetición del niño no es indiscriminada o sistemática sino selectiva, es decir, el niño se fija en aquellos aspectos propios de su etapa de desarrollo lingüístico. No es suficiente con que el niño oiga hablar en su lengua nativa. Para aprenderla, es preciso que hable con otros miembros de su comunidad lingüística, quienes le piden y le ofrecen aclaraciones o reajustes lingüísticos. Por tanto, se establece una negociación y retroalimentación (feedback) importantísimos para el progreso lingüístico de la L1 (Lightbown y Spada, 1993: 14). En cuanto a la acentuación, lo más probable es que los niños no aprendan la posición del acento en cada palabra, sino más bien una serie de reglas de acentuación. (Hochberg, 1988)

La adquisición del sistema de entonación en la L1 se presupone más compleja. Si bien hay un consenso generalizado en considerar la adquisición de la prosodia como previa a la de los sonidos, es decir, que la prosodia es el primer componente lingüístico que adquirimos en nuestra L1. No obstante, al hablar de adquisición, conviene puntualizar si se trata de capacidad de producción o sólo de percepción, si la adquisición de un aspecto determinado ya se ha completado o sólo han aparecido los primeros indicios, etc. Es cierto que a una edad temprana los niños ya tienen un cierto dominio de los entonemas o formas lingǘsticas de su L1, pero para la adquisición de la amplia gama de funciones asociadas a ellos son precisos varios años más (Cortés, 2001).

En definitiva, teniendo en cuenta los diferentes aspectos tratados, podemos señalar que la competencia prosódica se debe desarrollar en una comunidad lingüística por medio de interacciones comunicativas en las que los verbos «hablar» y «entender» se lleven a la práctica, pero las preguntas que debemos hacernos son: ¿se ha hecho algo para desarrollar la competencia prosódica de los alumnos? ¿Qué método se ha utilizado? ¿Por medio de qué técnica? 
Por tanto, el manejo de los aspectos prosódicos constituye uno de los primeros pasos en el desarrollo lingüístico del niño. Como se ha argumentado, la prosodia servirá de base para la expresión de determinadas intenciones comunicativas en momentos en los que el dominio léxico y segmental del niño no alcanza aún los estándares adultos.

\section{Corpus y metodología}

\subsection{Participantes}

Los sujetos de este estudio lo componen seis niños y niñas vascos con lengua materna euskera y, que en el estudio se identifican como MBR [masc.], ELB [masc.], ALL [masc.], ARL [fem.], LBG [fem.] y LBR [fem.]. Los niños pertenecen a hogares de clase media de un pequeño pueblo de la costa vizcaína, Bermeo, situado a 30 kilómetros al nordeste de Bilbao. Además, desde un punto de vista sociolingüístico, hay que señalar que Bermeo es un pueblo con una importante presencia de la lengua vasca, tal y como se puede comprobar en la web oficial de estadísticas del Gobierno Vasco (EUSTAT: http://www.eustat.es). En este contexto, los padres de los niños participantes hablan exclusivamente en vasco con los niños. Además, se dan dos parámetros más a tener en cuenta: los informantes eran, en el momento de las grabaciones, hijos únicos; y todavía no habían sido escolarizados en el primer ciclo de la Educación Infantil.

\subsection{Procedimiento}

El material empleado para inducir a los sujetos al juego fueron una serie de actividades de juego libre e interactuando con ellos su cuidador. Antes de comenzar la grabación, se les dijo a los cuidadores que jugaran con los niños verbalizando sus acciones, respondiendo, etc., tal como se hace normalmente en situaciones cotidianas de interacción. Las sesiones fueron grabadas en vídeo en las respectivas casas de los sujetos (habitaciones de los niños, cuarto de estar, etc.). El investigador sujetaba la cámara mientras se producía la interacción entre el niño y su cuidador, y se movía con la cámara detrás de ellos si se producían cambios de escenario. 
A este respecto, hay que señalar que la consulta de la bibliografía existente nos muestra que la presencia del adulto, conocido para el niño, influye de forma positiva en la calidad del material registrado (Siguán, 1983; Bruner, 1984; Man Shum, 1986; Boada, 1986, entre otros). La riqueza que surge de la interacción niño-adulto es quizás una consecuencia del valor intencional que inevitablemente poseen las intervenciones del adulto. Tanto en las grabaciones como en la vida diaria actúa como organizador de las interacciones, $y$, por supuesto, como modelo que marca pautas de aprendizaje. La presencia del adulto se constituye como un elemento habitual en el proceso adquisitivo. No hay que olvidar que para que éste se desarrolle con normalidad debe llegar a establecerse una “interacción íntima con adultos ya hablantes” (Clemente Estevan, 1998: 42).

Las sesiones de grabación tuvieron lugar cronológicamente durante una semana. La duración de las mismas era de 20 y 45 min. aproximadamente, dependiendo de la atención que mostraran los niños. Asimismo, hay que señalar que todas las grabaciones se han hecho públicas a través de la plataforma HAURNET, que incluye grabaciones de otros niños con perfiles similares. Las grabaciones se realizaron utilizando una cámara ZOOM modelo Q3HD. No se emplearon micrófonos adicionales por dos razones: en primer lugar, porque la propia cámara cuenta con excelente micrófono para la grabación de sonido estéreo tridimensional y de alta calidad; y en segundo lugar, porque desde un punto de vista metodológico al instalar micrófonos externos en la ropa de los niños o en el escenario de grabación se podía distorsionar el ambiente natural de grabación.

\subsection{Codificación}

En total, los niños fueron registrados en 9 sesiones con un cómputo total de unas 3 horas de grabación, aproximadamente. En la Tabla 1 encontramos la información del corpus al respecto. 
Tabla 1. Datos de los informantes

\begin{tabular}{|l|l|l|l|}
\hline $\begin{array}{l}\text { Siglas que identifican } \\
\text { al informante }\end{array}$ & $\begin{array}{l}\text { Edad de la } \\
\text { grabación }\end{array}$ & $\begin{array}{l}\text { Número de grabaciones } \\
\text { en las que participa }\end{array}$ & $\begin{array}{l}\text { Duración de todas } \\
\text { las grabaciones del } \\
\text { informante }\end{array}$ \\
\hline MBR [masc.] & $1 ; 01.20$ & 2 & $53^{\prime} 26^{\prime \prime}$ \\
\hline ELB [masc.] & $1 ; 08.17$ & 1 & $19^{\prime} 11^{\prime \prime}$ \\
\hline ALL [masc.] & $1 ; 07.2$ & 2 & 1 h. $01^{\prime} 09^{\prime}$ \\
\hline ARL [fem.] & $1 ; 3.23$ & 2 & $48^{\prime} 19^{\prime}$ \\
\hline LBG [fem.] & $1 ; 02.11$ & 1 & $21^{\prime} 11^{\prime \prime}$ \\
\hline LBR [fem.] & $1 ; 06.5$ & 2 & $35^{\prime} 17^{\prime \prime}$ \\
\hline
\end{tabular}

Asimismo, hay que señalar que este rango de edad fue seleccionado precisamente por tratarse del periodo clave en el que se producen los primeros actos comunicativos verbales intencionados en su transición desde el balbuceo variado (conversacional) al período lingüístico de una o dos palabras (formas fonéticas estables o consistentes si su criterio se centra en la forma sonora, o protopalabras, si hay interés de observar los aspectos intencionales y referenciales).

Todos los actos comunicativos producidos por los niños fueron identificados y localizados en las grabaciones, utilizando el software de transcripción multimodal ELAN (Lausberg \& Sloetjes, 2009). El análisis comprendió dos momentos principales, codificados y analizados mediante dos tiras de transcripción usando ELAN (Fig. 1): acto comunicativo (communicative act) y vocalización (speech). Así, las aproximadamente 3 horas de grabación fueron segmentadas en 675 vocalizaciones, de las cuales 522 se clasificaron como comunicativas y 153 fueron clasificadas como no comunicativas.

Con el objetivo de comprobar la fiabilidad de la ubicación de los actos comunicativos con las combinaciones anteriormente descritas, se utilizó el procedimiento de acuerdo inter-jueces con dos evaluadores externos e independientes y mediante el cálculo del índice Kappa (Cohen, 1960). El coeficiente de Kappa se utilizó en un subconjunto del 15\% de los datos (101 casos), dando como resultado un estadístico Kappa de 0,834, lo que indica que hubo un acuerdo sustancial entre codificadores con respecto a la identificación y localización de los actos comunicativos. En cuanto a la clasificación de 
los actos comunicativos en una de las dos categorías, el estadístico Kappa ha sido de 0,723, considerado también como un índice de acuerdo excelente.

En primer lugar, se ha identificado y codificado el acto comunicativo (communicative act) con las etiquetas acto comunicativo (communicative act) o acto no comunicativo (not communicative act) (ver tira 1 en Fig. 1). La acción del niño fue considerada como conducta intencionada comunicativa cuando se observaron los siguientes indicios, (a) el niño orienta su mirada hacia el adulto interlocutor, actual o potencial, mientras vocaliza; (b) el niño proporciona al adulto un tiempo para intervenir después de haber vocalizado; (c) el adulto se siente interpelado por la vocalización infantil y, en esa medida, responde o comenta la intervención vocal del niño; (d) el niño manifiesta su frustración cuando, aparentemente, la reacción del interlocutor no lo satisface y modifica su emisión vocal en consecuencia. En este punto, hay que señalar que la percepción de los adultos de los actos comunicativos de los bebes como intencionales ha sido ampliamente utilizado como medida predictora, a la hora de investigar el desarrollo de la lengua y las capacidades cognoscitivas en los niños (Feldman \& Reznick, 1996; Sacks, 1997; Rivero, 2003; Papaeliou \& Trevarthen, 2006; Rochat, 2007).

Figura 1. Imagen de ELAN con tiras de anotación

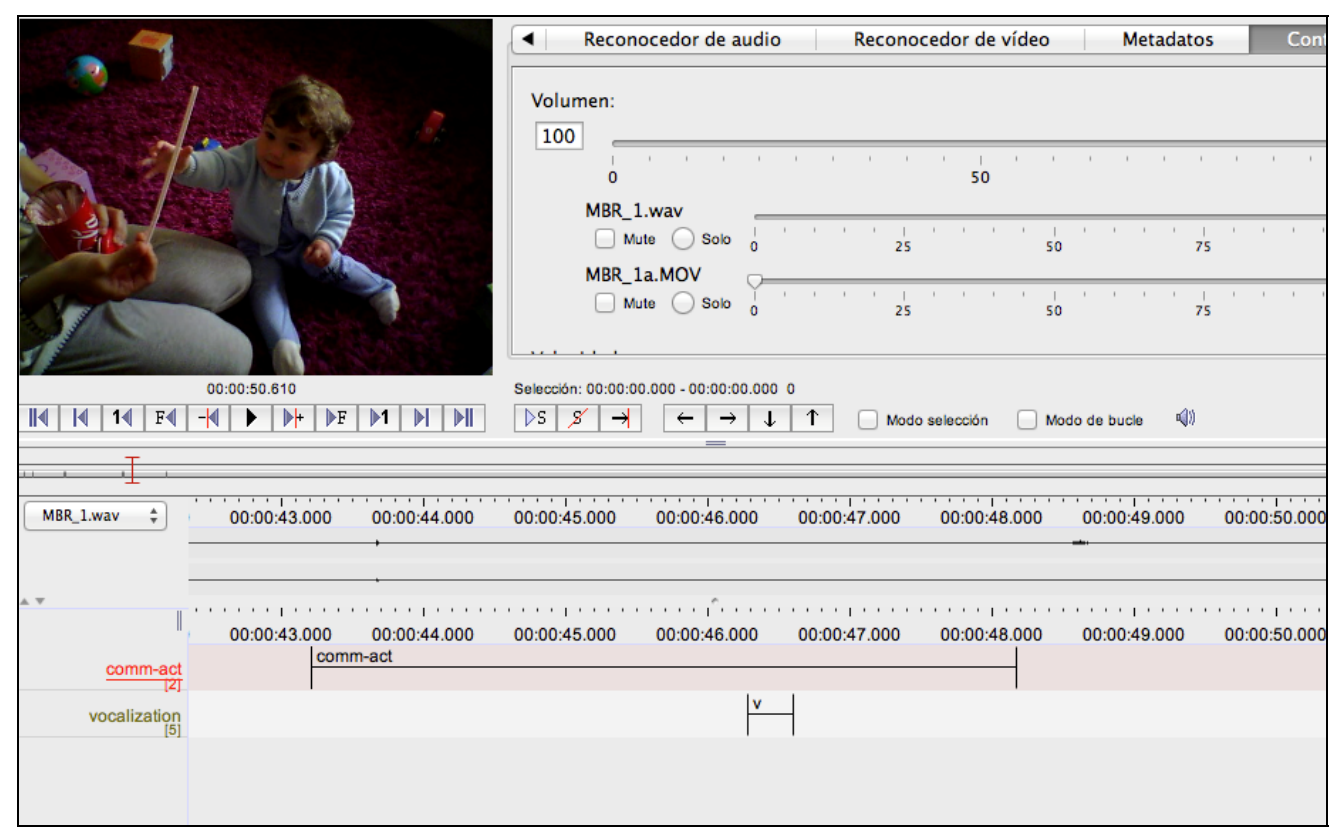


Esta categoría incluye cualquier vocalización producida por el niño, excepto los sonidos puramente "vegetativos”, o la risa, los gritos y el llanto. Se descartaron asimismo, las producciones emitidas por los niños teniendo un objeto en la boca (p. ej. el chupete). Por otra parte, desde un punto de vista pragmático tan sólo se codificaron las vocalizaciones producidas como un acto comunicativo intencional; es decir, sólo se incluyeron como vocalizaciones las que claramente se refieren al estímulo objetivo y las que se dirigen al adulto después de que el niño haya visto el estímulo (Goldstein \& Pollock, 2010). En cuanto a la delimitación exacta de la vocalización, se utilizó el criterio de codificar una vocalización como expresión independiente cuando había una separación superior a los 300 ms. (Igualada, Bosch \& Prieto, 2015; Oller, Wieman, Doyle \& Ross, 1976).

En segundo lugar, se determinó la función de las vocalizaciones comunicativas (ver Tabla 2). Para la codificación de las funciones vocálicas se establecieron las siguientes categorías, atendiendo a la taxonomía de Karousou (2003): función declarativa (declarative) el niño llama la atención del interlocutor sobre un determinado objeto, acción (o evento) o persona a través de vocalizaciones que refieren de forma explícita a éstos; función imperativa (imperative) el niño vocaliza con la intención de obtener algo del interlocutor: que le entregue un objeto, que realice una determinada acción, que le proporcione cierta información o que le preste atención; función expresiva (emotive) el niño expresa su reacción emocional ante una iniciativa del adulto interlocutor, que busca reforzar o evitar según le agrade o no. Esta emoción puede ser de protesta, rechazo, agrado o satisfacción, y está dirigida al interlocutor; función mímica (mimic) el niño reproduce las características segmentales o suprasegmentales de un enunciado anterior reciente del adulto, aunque sus vocalizaciones no reproducen la función con la que éste fue originalmente producido por el adulto; función guía de acción (action guide) el niño orienta u ordena su propia acción sin dejar de dirigirse al interlocutor por medio de estas vocalizaciones, que produce como acompañamiento de una acción habitual que está llevando a cabo; y la función fática (fatica) el niño responde a preguntas, sugerencias u órdenes del interlocutor con vocalizaciones que le permiten ocupar el turno en la conversación correspondiente, con el objetivo de mantener "vivo" el intercambio conversacional (ver tira 2 en Fig. 1). 
Tabla 2. Sumario de funciones acompañado de imágenes

\begin{tabular}{|l|l|}
\hline Tipo de función & Imagen \\
\hline Declarativa & \\
\hline Imperativa & \\
\hline Mímica & \\
\hline Emotiva & \\
\hline
\end{tabular}

Al igual que en los casos anteriores, con el objetivo de comprobar la fiabilidad de la codificación de la función de la vocalización, se utilizó el valor estadístico Kappa de Cohem con los dos evaluadores independientes que participaron anteriormente. En este caso, el coeficiente de Kappa se utilizó en un subconjunto arbitrario del 20\% de los datos (104 casos), dando como resultado un estadístico Kappa de 0,823 , lo que indica que hubo un acuerdo sustancial entre codificadores con respecto a la identificación de la función vocálica.

Finalmente, mediante la opción de exportar datos de ELAN, se analizaron los audios correspondientes a todas las vocalizaciones -comunicativas y no comunicativas con el programa Praat (Boersma \& Weenink, 2015). Por tanto, la codificación prosódica se 
realizó mediante el programa Praat y utilizando el modelo AM (Beckman \& Pierrehumbert, 1986; Hualde, 2003; Ladd, 2008, entre otros). El modelo AM concibe las curvas melódicas como una concatenación lineal de dos clases de unidades fonológicas con propiedades distintas: los acentos tonales (pitch accents), o movimientos tonales que se asocian con sílabas acentuadas y los tonos de frontera (boundary tones), o movimientos tonales que se asocian con límites prosódicos. El modelo propone una versión radical del análisis por niveles y defiende que los contornos se pueden representar adecuadamente utilizando sólo dos niveles tonales, el alto (H) y el bajo (L). Los tonos H o L se marcan con el diacrítico estrella * para indicar su asociación con las sílabas acentuadas y con el diacrítico porcentaje \% para indicar su asociación con la frontera de un constituyente prosódico. En esta investigación se han analizado los tonos de frontera siguiendo el esquema que se recoge en la Tabla 3.

Tabla 3. Diferencias entre los distintos contornos entonativos

\begin{tabular}{|l|l|l|}
\hline H\% & $\begin{array}{l}\text { H\% para los casos de subida de F0 } \\
\text { desde un tono bajo anterior } \\
\text { (izquierda) o continuación de F0 } \\
\text { desde un tono alto anterior } \\
\text { (derecha). }\end{array}$ \\
\hline LH\% & $\begin{array}{l}\text { LH\% descenso-ascenso de F0 } \\
\text { después de un tono alto (izquierda) } \\
\text { o F0 baja con subida posterior si el } \\
\text { tono anterior es bajo (derecha). }\end{array}$ \\
\hline HL\% & $\begin{array}{l}\text { L\% bajada de F0 desde un tono } \\
\text { alto anterior (izquierda) o F0 baja } \\
\text { desde un tono bajo (derecha). }\end{array}$ \\
\hline
\end{tabular}


En la Figura 2 se presenta el ejemplo de transcripción ortográfica y prosódica realizado con Praat para la palabra ama 'mamá' producida por ARL en 1; 3. Con todo, hay que señalar que sólo se tomaron en consideración -por contener una vocalización- los sonidos del habla con propósito comunicativo; en todo caso, palabras en vasco utilizadas de forma coherente. En cuanto a la codificación, (a) en la primera línea se presenta la transcripción ortográfica de la vocalización en sus puntos inicial y final; (b) en la segunda línea se describe la transcripción fonética de las sílabas; (c) y en la tercera línea se analiza los límites de la prominencia prosódica. En el caso de que la sílaba acentuada de la vocalización o la palabra no estaba claramente identificada, se ha codificado como una categoría extra llamada sílaba acentuada difusa y, lógicamente, excluida de los análisis estadísticos ( $\mathrm{N}=138)$.

En el esquema se describe a continuación, se describe el procedimiento seguido para la codificación de la vocalización.

1. Localización del acto comunicativo portador de la vocalización.

2. Localización de los límites temporales de la silabificación.

3. Descripción y codificación de la prominencia prosódica

Figura 2. Ejemplo del etiquetado acústico en Praat para la palabra ama (mamá)

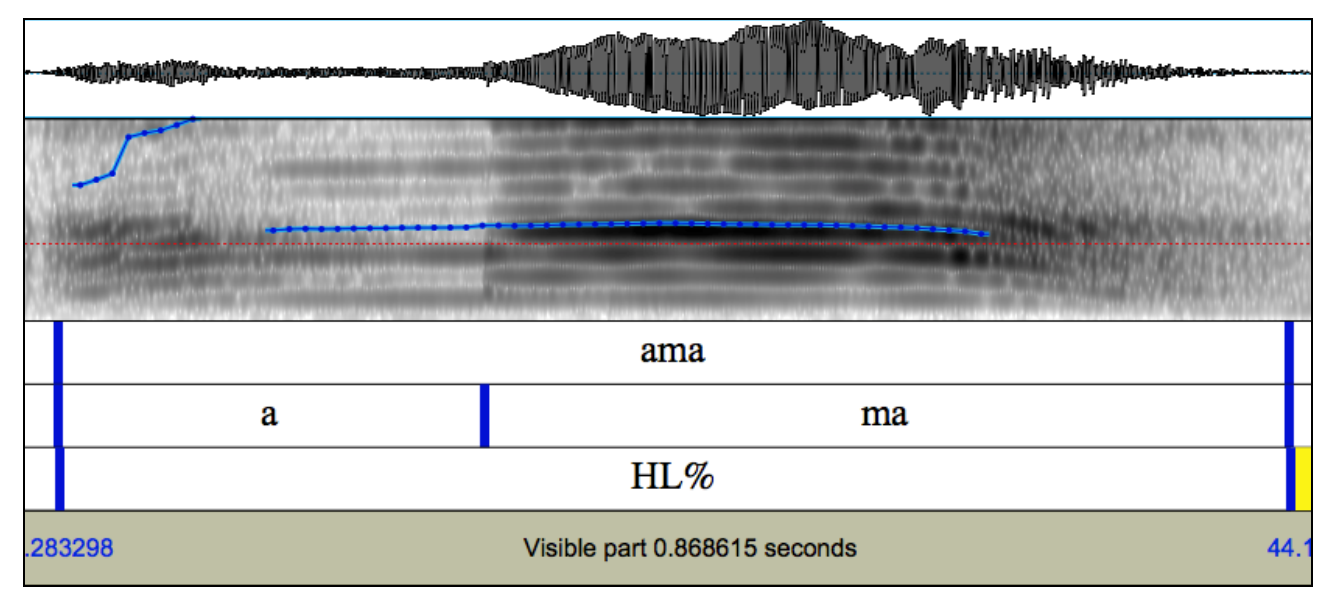

3. Resultados de la investigación

Del total de 675 vocalizaciones (comunicativas y no comunicativas), tan sólo en 38 (5,62\%) de ellas resultó imposible determinar la dirección final del contorno entonativo debido en su mayor parte al ruido ambiental o superposición de voces. Además, no se 
pudo establecer la función en 41 (7,85\%) vocalizaciones comunicativas por falta de elementos contextuales suficientes.

En primer lugar, para probar si había una significación estadística en la variación entre ‘vocalización comunicativa' y ‘vocalización no comunicativa' en relación con el contorno entonativo final de las vocalizaciones, se utilizó la prueba Chi-cuadrado de independencia (ver Tabla 4).

Tabla 4. Relación entre presencia/ausencia de intención comunicativa y contorno entonativo

\begin{tabular}{|l|l|l|l|l|}
\hline & $\mathrm{N}$ & Descendente & Ascendente & Total \\
\hline Comunicativas & 497 & 72,8 & 27,2 & 100 \\
\hline No comunicativas & 140 & 67,5 & 32,5 & 100 \\
\hline Total & 637 & \multicolumn{3}{|l}{} \\
\hline
\end{tabular}

Los resultados no mostraron significación estadística $\chi^{2}(2, \mathrm{~N}=637)=0.873, p$ $=0.658$ ). Por tanto, no es posible distinguir las vocalizaciones comunicativas de las no comunicativas a partir de un contorno final entonativo, por lo que no hay una relación entre ambas y su estudio exigiría un análisis autónomo.

En relación con la segunda cuestión planteada, es decir, qué tipo de relación se produce entre el 'contorno entonativo final' y las ‘funciones comunicativas específicas’, la Tabla 4 muestra la distribución correspondiente a la tipología funcional (expresada en números absolutos y porcentajes) para todas las posibilidades entonativas analizadas. Los resultados muestran como el tono descendente -en sus dos variantes L\% y HL\%- es el más común. Hay que destacar que el porcentaje más alto de tonos ascendentes se halla en las funciones imperativas (H\%: 25.51 y LH\%: 26) y los porcentajes más altos de tonos descendentes se encuentran en las funciones declarativas (L\%: 29.26) y funciones mímicas (HL\%: 33). 
Tabla 5. Número total y porcentual del contorno entonativo en combinación con la función

\begin{tabular}{|l|l|l|l|l|l|l|l|l|l|l|l|l|l|}
\hline \multirow{2}{*}{ Tono } & \multicolumn{2}{l|}{ Imperativa } & \multicolumn{2}{l|}{ Declarativa } & \multicolumn{2}{l|}{ Emotiva } & \multicolumn{2}{l|}{ Mímica } & \multicolumn{2}{l|}{ Guía } & \multicolumn{2}{l|}{ Fática } \\
\cline { 2 - 15 } & $N$ & $\%$ & $N$ & $\%$ & $N$ & $\%$ & $N$ & $\%$ & $N$ & $\%$ & $N$ & $\%$ \\
\hline H\% & 25 & 25.51 & 8 & 8.16 & 12 & 12.24 & 16 & 16.32 & 21 & 21.42 & 16 & 16.32 \\
\hline LH\% & 13 & 26 & 6 & 12 & 4 & 8 & 7 & 14 & 9 & 18 & 11 & 22 \\
\hline L\% & 36 & 17.56 & 60 & 29.26 & 22 & 10.73 & 45 & 21.95 & 19 & 9.26 & 23 & 11.21 \\
\hline HL\% & 16 & 15.53 & 26 & 25.24 & 9 & 8.73 & 34 & 33 & 12 & 11.65 & 6 & 5.82 \\
\hline
\end{tabular}

En cuanto a la tipología funcional, si realizamos un desglose por tipo de función y su correspondencia tonal, se observa con interés como la función imperativa se transmite con una proporción mayoritaria por medio de la tonalidad H\% y LH\%, mientras que las tonalidades L\% y HL\% se distribuyen mayoritariamente pero significativamente entre las funciones L\% y HL\% (ver Figura 3).

Figura 3. Ratio de distribución de porcentajes según la correspondencia función/tipología tonal

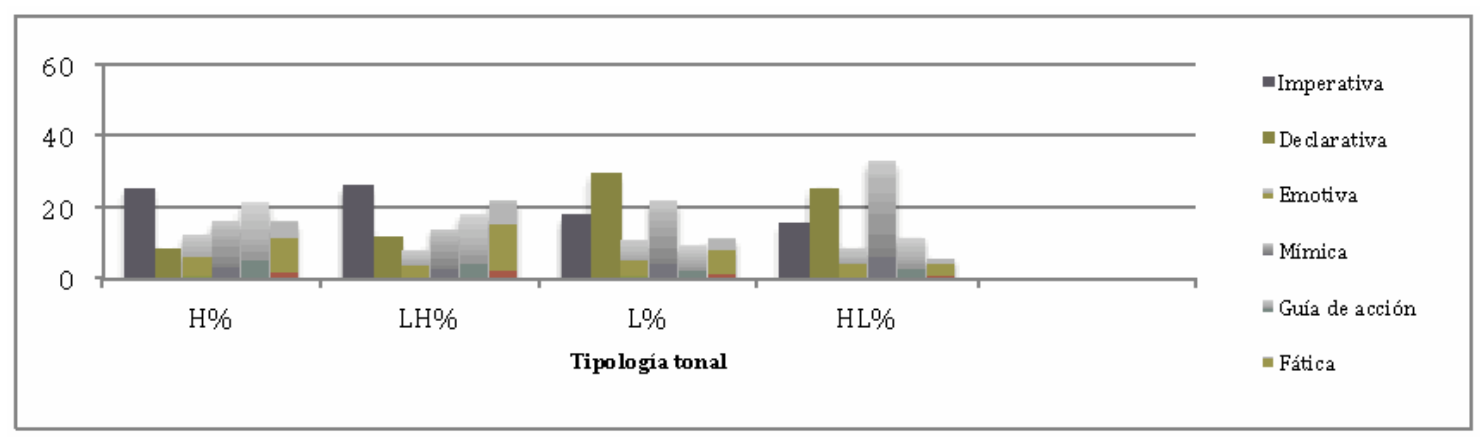

Con el objetivo de probar si había una significación estadística en la variación entre el ‘contorno entonativo final' y las ‘funciones comunicativas específicas’ se utilizó la prueba Chi-cuadrado de independencia. Los resultados mostraron que había una significación estadística para la tonalidad descendente $\left(\chi^{2}(2,22.365, p<.001)\right.$ sobre las ascendentes. Por tanto, aunque no hay una función que se caracterice por un uso mayoritario del tono ascendente, este sí es predominante en determinadas funciones; tal y como se ha señalado en el caso de la función imperativa. Ahora bien, se trata tan sólo de una diferenciación relativa sin significación estadística.

El desarrollo precoz de la entonación ya en el período de una sola palabra se constata en la aparición de tonos de frontera complejos durante esta etapa. ELB produce contornos entonativos complejos $\mathrm{L}+\mathrm{H}^{*} \mathrm{LH} \%$ y $\mathrm{L}+\mathrm{H}^{*} \mathrm{HL} \%$ antes de la producción combinatoria de dos palabras en 1; 8. Así, por ejemplo, la Fig. 2 muestra el patrón 
entonativo de la vocalización ama 'mamá' producida por ARL en 1; 3. En el contexto registrado, este contorno entonativo tiene la función de llamar la atención de su madre. Así, la sílaba acentuada con tono ascendente se conjuga con el tono de frontera complejo ascendente-descendente.

La Figura 4 representa el número de diferentes tipos de configuración tonal registrados en los niños/as seleccionados. Esta figura demostra claramente: (1) todos los niños producen dos o tres modalidades entonativas distintas desde la aparición de la vocalización/palabra; y (2) la edad en que se realizó la grabación está relacionada con la progresión cuantitativa en torno a los diferentes configuraciones tonales. Así, de las escasas tres configuraciones registradas en MBR 1;1 pasamos a las más de 200 descritas para ELB 1;8. A tenor de estos resultados, parece demostrarse que hay un importante salto cuantitativo en el desarrollo de la tipología entonativa a partir de los 18 meses. Ahora bien, para justificar metodológicamente esta afirmación sería necesario realizar una investigación longitudinal en estos mismos niños y observar el aumento entonativo atendiendo a su progresión cronológica. En este punto son muy interesantes los datos constatados para el catalán por Prieto et al. (2011).

Figura 4. Ratio de distribución de los contornos entonativos producidos por los niños/as analizados

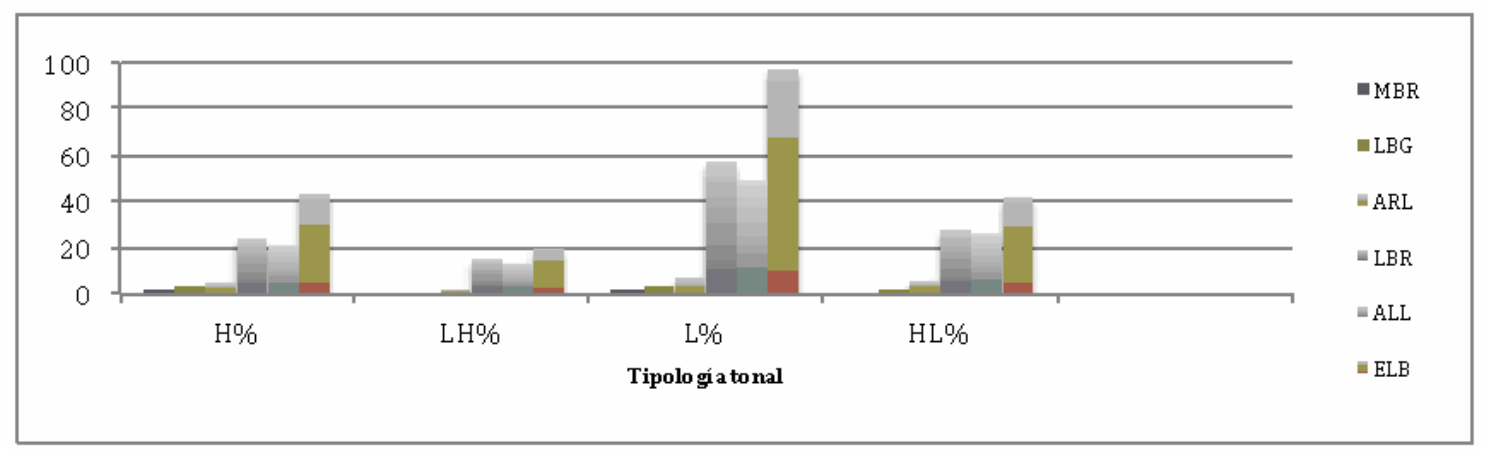

\section{Conclusiones}

En primer lugar, hay que señalar que las vocalizaciones comunicativas y no comunicativas no se diferencian significativamente en cuanto a su contorno entonativo final. Lógicamente, esta ausencia de relación podría deberse a la diferencia cuantitativa entre las muestras de vocalizaciones comunicativas y no comunicativas, ya que estas 
últimas son bastante más escasa, por lo que sería conveniente en investigaciones futuras incrementar este número con el objeto de afianzar nuestra afirmación.

En segundo lugar, hay que destacar que las funciones comunicativas consideradas en este estudio se diferencian de forma significativa por su contorno entonativo final. Así, es evidente el predominio del contorno descendente, datos que concuerdan con la propuesta de Lieberman (1984) o con el planteamiento teórico de Quilis (1993), que relaciona el predominio del tono final descendente como una sintonización con el uso prosódico presente en el entorno, en tanto en cuanto el tono descendente es el característico de los enunciados declarativos en el español adulto.

Así mismo, se confirma la predominancia relativa de la tonalidad ascendente para el caso de la función imperativa, circunstancia ya señalada para el inglés por Marcos (1987) y Flax (1991) y para el español por Fernández (2014). Además, según los resultados, hay ciertas funciones en las que el tono ascendente se usa más que en otras, aunque en ningún caso superaría la proporción total de tonos descendentes. Con todo, no se halló ninguna función comunicativa que se expresará únicamente por medio de un único tono, ni un tono que se asociara exclusivamente a una única función. Esta circunstancia se podría relacionar con investigaciones recientes, en las que se confirma la coordinación temporal entre gesto deíctico de señalar y alcanzar con una motivación imperativa y la producción vocal, ya que en estos casos el gesto deíctico necesitaba de más tiempo para su ejecución, con lo que se podría coordinar de una manera más adecuada con la vocalización (Esteve-Gibert et al., 2014; Esteve-Gibert et al., 2015; Romero, 2015). Esto podría avalar la hipótesis de que otros elementos junto con la prosodia -como el gesto- ofrecerían una información importante al niño para solicitar de su interlocutor una intención comunicativa asociada a su vocalización.

Por otra parte, es interesante resaltar que los porcentajes más altos de tonos ascendentes recaen principalmente en las funciones imperativa, fática y guía de acción. La presencia mayoritaria de la función imperativa podría deberse a la necesidad de buscar una reacción en el interlocutor o a vocalizaciones que demandan información por parte del destinatario, lo que contribuiría a la utilización del tono final ascendente. La evolución de esta función fática paralela al desarrollo cronológico evidenciaría una comprensión del diálogo como construcción cooperativa en la que prima la interacción, la alternancia de turnos, más allá de la capacidad para producir un enunciado de respuesta 
verdaderamente relevante; es decir, la voluntad de interpelar más enfáticamente al adulto, y por tanto postura tradicionalmente asociada al tono ascendente. Podríamos incluso intuir aquí el funcionamiento del principio de cooperación de Grice (1975), en tanto la conducta del niño parece revelar la conciencia de que, ante ciertos enunciados del adulto, él se encuentra obligado a cooperar produciendo, a su vez, una emisión vocal en respuesta. Esta circunstancia se ve avalada por el aumento porcentual que se produce en las funciones mímica y guía de acción, como funciones significativas del interés del niño de participar y permanecer en la interacción con el adulto.

Además, la presencia minoritaria -en términos porcentuales- de la función declarativa asociada a los tonos descendentes, se explicaría por un cambio de objetivo, es decir, la función declarativa se centra en el referente -declarar algo acerca de un objeto, por ejemplo- y no tanto en la interpelación al destinatario del mensaje. Sin embargo, somos conscientes de las limitaciones de este estudio transversal sobre 675 vocalizaciones entre seis niños en un contexto de observación natural libre. Así, en investigaciones futuras se debería incrementar este número de informantes a través de un estudio longitudinal con el objeto de afianzar nuestra afirmación de que las funciones comunicativas consideradas en este estudio se diferencian de forma significativa por su contorno entonativo final.

Por tanto, los resultados indican que los seis niños analizados producen contornos entonativos complejos ya desde el inicio de la primera palabra, y que esta variación se va haciendo cada vez más numerosa y compleja con la edad, lo que nos puede llevar a interpretar que los niños demuestran un conocimiento importante de la gramática entonativa del adulto. Es importante destacar que nuestros datos niños muestran patrones entonativos relacionados adecuadamente con la forma pragmática que desean emplear para comunicarse con el interlocutor. Datos recientes nos hacen relacionar estos datos con otras descritos para otras lenguas: catalán, portugués europeo o holandés, por ejemplo (Prieto et. al., 2011; Chen \& Fikkert, 2007; Frota \& Vigário, 2008).

Los niños seleccionados en este estudio demuestran un dominio en la utilización del acento tonal y de los tonos de frontera ya en el comienzo del habla y, además como se ha visto, una evolución importante en la producción de patrones entonativos, cada vez más complejos. Además, el estudio demuestra la validez del método autosegmental aplicado en adultos para la realización y descripción de inventarios de acentos tonales y 
combinaciones de tonos de frontera-, en la aplicación y análisis de los patrones entonativos producidos por bebés con vasco como lengua materna. Lógicamente, se ve la necesidad de realizar estudios longitudinales futuros con la ampliación al periodo de dos palabras, con el objeto de poder realizar análisis contrastivos con los inventarios de contorno entonativo realizados en vasco para esta zona septentrional de Bizkaia (Gaminde et al. 2012).

Bibliografía

Baeza-Álvarez, R.L.; Rodríguez-Maldonado, D.D. (2011). Comprensión de la prosodia en el lenguaje verbal de niños escolares. Revista de Educación y Desarrollo, 17, 5-14.

Bates, E., Camaioni, L. \& Volterra, V. (1975). The acquisition of performatives prior to speech. Merrill-Palmer Quarterly of Behavior and Development, 21, 205-224.

Beckman, M. \& Pierrehumbert, J.B. (1986). Intonational structure in English and Japanese. Phonology Yearbook, 3, 255-310.

Bever, T.G.; Fodor, J.A.; Weksel, W. (1971). Theoretical notes on the acquisition of syntax: a critique of contextual generalization. En A. Bar-Adon, W.F. Leopold (Eds.). Child Language: A Book of Readings. Englewood Cliffs: Prentice-Hall.

Boersma, P. \& Weenink, D. (2015). Praat: doing phonetics by computer [Computer program]. Version 5.4.22. http://www.fon.hum.uva.nl/praat/

Boysson-Bardies, B. de; Bacri, N., Sagart, L. \& Poizat, M. (1981). Timing in late babbling. Journal of Child Language, 8(3), 525-539.

Boysson-Bardies, B. de \& Vihman, M.M. (1991). Adaptation to language: Evidence from babbling and first words in four languages. Language, 67, 297-319.

Butcher, C. \& Goldin-Meadow, S. (2000). Gesture and the transition from one- to twoword speech: when hand and mouth come together. En D. McNeill (Ed.), Language and Gesture (pp. 235-258). New York: Cambridge University Press.

Chen, L.M. \& Fikkert, P. (2007). Intonation of early two-word utterances in Dutch. En J. Trouvain \& W.J. Barry (Eds.), Proceedings of the XVIth International Congress of Phonetic Sciencies (pp. 315-320). Pirrot GmbH: Dudweiler. 
Clemente Estevan, R.A. (1998). El papel del adulto en la adquisición del lenguaje. Reflexiones sobre los valores diferenciales entre interlocutores. En M. Peralbo Uzquiano et al. (Eds.), Desarrollo del lenguaje y cognición (pp. 41-52). Madrid: Pirámide.

Coggins, T.E. \& Carpenter, R.L. (1981). The Communicative Intention Inventory: A system for observing and coding children's early intentional communication. Applied Psycholinguistics, 2, 235-251.

Cohen, J. (1960). A coefficient of agreement for nominal scales. Educational and Psychological Measurement, 20 (1), 37-46.

Cortés, M., (2001): Producción y adquisición de la entonación española en enunciados de habla espontánea: el caso de los estudiantes taiwaneses. Estudios de Fonética Experimental, 11, 89-119.

D’Odorico, L. (1984). Non-segmental features in pre-linguistic communications: an analysis of some types of infant cry and non-cry vocalizations. Journal of Child Language, 11 (1), 17-27.

D’Odorico, L. \& Franco, F. (1991). Selective production of vocalization types in different communication contexts. Journal of Child Language, 18 (3), 475-499.

Dore, J. (1974). A pragmatic description of early language development. Journal of Psycholinguistic Research, 3 (4), 343-350.

Dore, J. (1975). Holophrases, speech acts and language universals. Journal of Child Language, 18, 3-19.

Elbers, L. (1982). Operating principles in repetitive babbling: A cognitive continuity approach. Cognition, 12, 45-63.

Esteve-Gibert, N. \& Prieto P. (2014). Infants temporally coordinate gesture-speech combinations before they produce their first words. Speech Communication, 57, 301-316. Doi:10.1016/j.specom.2013.06.006

Esteve-Gibert, N., Prieto, P. \& Pons, F. (2015). Nine-month-old infants are sensitive to the temporal alignment of prosodic and gesture prominences. Infant Behavior and Development 38, 126-129. Doi:10.1016/j.infbeh.2014.12.016

Feldman, R. \& Reznick, J.S. (1996). Maternal perception of infant intentionality at 4 and 8 months. Infant Behavior and Development, 19, 483-496. 
Fernández Flecha, M.A. (2014). La adquisición de las relaciones entre prosodia e intención comunicativa: primeras asociaciones entre forma y función. Lexis, 38 (1), 5-33.

Flax, J.; Lahey, M.; Harris, K. \& Boothroyd, A. (1991). Relations between prosodic variables and communicative functions. Journal of Child Language, 18, 3-19.

Frota, S. \& Vigário, M. (2008). The intonation of one-word and first two-word utterances in European Portuguese. Paper presented at the Third Conference on Tone and Intonation (TIE 3), Lisboa, 15-17 septiembre 2008.

Gaminde, I.; Romero, A. \& Legarra, H. (2012). Gramatika eta hizkuntza bariazioa Bermeon. Bermeo: Erroteta.

Goldstein, B.A. \& Pollock, K.E. (2000). Vowel errors in Spanish-speaking children with phonological disorders: a retrospective, comparative study. Clinical Linguistics and Phonetics, 14, 217-225.

Grice, H.P. (1975). Logic and conversation. En P. Cole \& J.L: Morgan (Eds.), Syntax and Semantics. New York: Academic Press.

Halliday, M.A.K. (1982). Aprendiendo a conferir significado. En E.H. Lenneberg \& E. Lenneberg (Eds.), Fundamentos del desarrollo del lenguaje (pp. 239-275). Madrid: Alianza Universidad Textos.

Hualde, J.I. (2003). El modelo métrico y autosegmental. En P. Prieto (coord..), Teorías de la entonación. Barcelona: Ariel.

Igualada, A., Bosch, L. \& Prieto P. (2015). Language development at 18 months is related tomultimodal communicative strategies at 12 months. Infant Behavior and Development, 39, 42-52. Doi:10.1016/j.infbeh.2015.02.004

Juszyck, P.W. (1997). The discovery of spoken language. Cambridge: MIT Press.

Karousou, A. (2003). Análisis de las vocalizaciones tempranas: su patrón evolutivo y su función determinante en la emergencia de la palabra. Unpublished doctoral dissertation. Madrid: Universidad Complutense.

Kaye, K. (1982). The mental and social life of babies. Chicago: The University of Chicago Press.

Koopmans-van Beinum, F.J. \& Van der Stelt, J.M: (1986). Early stages in the development of speech movements. En B. Lindblom \& R. Zetterström (Eds.), Precursors of Early Speech (pp. 37-50). New York: Stockton Press. 
Ladd, D.R. (2008). Intonational phonology. Cambridge: Cambridge University Press. Lausberg, H. \& Sloetjes, H. (2009). Coding gestural behavior with the NEUROGESELAN system. Behavior Research Methods, 41 (3), 841-849.

Lightbown, P. M. \& Spada, N. (1990). Focus-on-form and corrective feedback in communicative language teaching: Effects on second language learning. Studies in Second Language Acquisition, 12, 429-448.

Lieberman, P. (1984). The Biology and Evolution of Language. Cambridge, MA: Harvard University Press.

Locke, J.L: (1983). Phonological Acquisition and Change. New York: Academic Press. López-Ornat, S. (1999): La adquisición del lenguaje. En F. Cuestos \& M. De Vega (coords.), Psicolingüística del español (pp. 78-98). Madrid: Trotta.

López Ornat, S. \& Karousou, A. (2005). Las Vocalizaciones tempranas (8-30 meses) y su relación con el vocabulario y la gramática. Su medida en el CDI-Español: Resultados preliminares. En M.A. Mayor Cinca, B. Zubiauz de Pedro \& E. DíezViñaloria (Eds), Estudios sobre la adquisición del lenguaje (pp. 401-420). Salamanca: Universidad de Salamanca.

MacNeill, D. (1992). Hand and Mind What Gestures Reveal about Thought. Chicago: University of Chicago Press.

Marcos, H. (1987). Communicative functions of pitch range and pitch direction in infants. Journal of Child Language, 14, 255-268.

Morgan, J.L.; Demuth, K. (eds.) (1996): Signal to Syntax: Bootstraping From Speech to Grammar in Early Acquisition. New York: L. Erlbaum Associates.

Murillo, E. \& Capilla, A. (2015). Properties of vocalization- and gesture- combinations in the transition to first words. Journal Child Language, 30, 1-24. Doi:10.1017/S0305000915000343

Nathani, S. \& Oller, D.K. (2001). Beyond ba-ba ang gu-gu: Challenges and strategies in coding infant vocalizations. Behavior Research Methods, Instruments, and Computers, 33, 3, 321-330.

Oller, D.K. (2000). The Emergence of the Speech Capacity. Mahwah, NJ,: Lawrence Erlbaum Associates, Inc., Publishers.

Oller, D.K., Wieman, L.A., Doyle, W.J. \& Ross, C. (1976). Infant babbling and speech. Journal of Child Language, 3, 1-11. 
Papaeliou, C.; Minadakis, G. \& Cavouras, D. (2002). Acoustic Patterns of Infant Vocalizations Expressing Emotions and Communicative Functions. Journal of Speech, Language, and Hearing Research, 45, 311-317.

Papaeliou, C. \& Trevarthen, C. (2006). Prelinguistic pitch patterns expressing ‘communication’ and ‘apprehension’. Journal of Child Language, 3 (3), 163-178.

Peña-Garay, M. (2005). Habilidades lingüísticas de los niños menores de un año. Revista de Neurología, 41(5), 291-298.

Piaget, J. (1936). La naissance de l'intelligence chez l'enfant. Neuchâtel: Délachaux et Niestlé, Traducción castellano, Madrid, Aguilar, 1969.

Prieto, P. \& Vanrell, M.M. (2007). Early intonational development in Catalan. En J. Trouvain \& W.J. Barry (Eds.), Proceedings of the XVIth International Congress of Phonetic Sciencies (pp. 309-314). Dudweiler: Pirrot GmbH.

Prieto, P.; Estrella, A. \& Thorson, J. (2011). Is prosodic development correlated with gramatical and lexical development? Evidence from emerging intonation in Catalan and Spanish. Journal Child Language, 39(2), 1-37. Doi: 10.1017/S030500091100002X

Quilis, A. (1993). Tratado de fonología y fonética española. Madrid: Gredos.

Rivero, M. (2003). Los inicios de la comunicación: la intencionalidad comunicativa y el significado como procesos graduales. Anuario de Psicología, 34 (3), 337-356.

Rochat, P. (2007). Intentional action arises from early reciprocal exchanges. Acta Psychologica, 124, 8-25. Doi:10.1016/j.actpsy.2006.09.004

Romero, A.; Etxebarria, A.; Gaminde, I. \& Garay, U. (2015). El papel de la prosodia en la enseñanza de la L1. Un aporte didáctico para el aula de Educación Infantil y de Educación Primaria. Revista Phonica, 11, 64-86.

Romero, A.; De Pablo, I.; Etxebarria, A. \& Romero, A. (2017). Interrelación entre gestos y vocalizaciones en funciones comunicativas tempranas: evidencias desde la lengua vasca. Revista Signos (en prensa).

Sacks, J. (1997). Communication development in infancy. En J. Berko-Gleason, The development of Language. Needham Heights, Allyn y Bacon.

Sarriá, E. (1991). Observación de la comunicación intencional preverbal: Un sistema de codificación basado en el concepto de la categoría natural. Psicotema, 3, 359-380. 
Snow, D. \& Balog, H.L. (2002). Do children produce the melody before the words? A review of development intonation research. Lingua, 112, 1025-1058.

Snow, D. (2004). Falling intonation in the one -and two-syllable utterances of infants and preschoolers. Journal of Phonetics, 32, 373-393. Doi.org/10.1016/S00954470(03)00038-X

Vihman, M.M. (1994). When is a word a Word? Journal of Child Language, 21, 517542.

Vihman, M.M. (1996). Phonological Development: The origins of language in child. Oxford, UK: Blackwell Publishers.

Vygotsky, L.S. (1979). Internalización de las funciones psicológicas superiores. En L.S. Vygotski, El desarrollo de los procesos psicológicos superiores. Barcelona: Crítica.

Waterson, N. (1981): A tentative developmental model of phonological representation. En T. Myers, J. Laver \& J. Anderson (Eds.). The cognitive representation of speech (23-56). Amsterdam: North Holland.

Recibido: 30 de octubre de 2015

Aceptado: 23 de noviembre de 2016

Publicado: 30 de noviembre de 2016

Actualizado: 5 de diciembre de 2016 